\title{
Hyperactivity of factor IX Padua (R338L) depends on factor VIIla cofactor activity
}

\author{
Benjamin J. Samelson-Jones, ${ }^{1,2,3}$ Jonathan D. Finn, ${ }^{1}$ Lindsey A. George, ${ }^{1,2,3}$ Rodney M. Camire, ${ }^{1,2,3}$ \\ and Valder R. Arruda $a^{1,2,3}$ \\ 1The Children's Hospital of Philadelphia, Philadelphia, Pennsylvania, USA. ${ }^{2}$ Perelman School of Medicine, University \\ of Pennsylvania, Philadelphia, Pennsylvania, USA. ${ }^{3}$ Raymond G. Perelman Center for Cellular and Molecular \\ Therapeutics, Philadelphia, Pennsylvania, USA.
}

\begin{abstract}
Adeno-associated virus (AAV) vector liver-directed gene therapy (GT) for hemophilia B (HB) is limited by a vector dose-dependent hepatotoxicity. Recently, this obstacle has been partially circumvented by the use of a hyperactive factor IX (FIX) variant, R338L (Padua), which has an 8-fold increased specific activity compared with FIX-WT. FIX-R338L has emerged as the standard for HB GT. However, the underlying mechanism of its hyperactivity is undefined; as such, safety concerns of unregulated coagulation and the potential for thrombotic complications have not been fully addressed. To this end, we evaluated the enzymatic and clotting activity as well as the activation, inactivation, and cofactor dependence of FIX-R338L relative to FIX-WT. We observed that the high specific activity of FIX-R338L requires factor VIIla (FVIIIa) cofactor. In a potentially novel system using emicizumab, an FVIII-mimicking bispecific antibody, the hyperactivity of both recombinant FIX-R338L and AAV-mediated transgene-expressed FIX-R338L from HB CT subjects was ablated without FVIIla activity. We conclude that the molecular regulation of activation, inactivation, and cofactor dependence of FIX-R338L is similar to FIX-WT but that the FVIIIadependent hyperactivity of FIX-R338L is the result of a faster rate of factor $X$ activation. This mechanism helps mitigate safety concerns of unregulated coagulation and supports the expanded use of FIX-R338L in HB therapy.
\end{abstract}

Conflict of interest: RMC and VRA receive research support and consulting fees from Pfizer. B|SJ and LAG are clinical investigators for gene therapy trials sponsored by Spark Therapeutics.

Copyright: @ 2019 American Society for Clinical Investigation

Submitted: March 7, 2019

Accepted: June 13, 2019

Published: June 20, 2019

Reference information: /CI Insight. 2019;4(14):e128683. https://doi. org/10.1172/jici.insight.128683.

\section{Introduction}

Hemophilia B (HB) is an X-linked bleeding disorder resulting from an inheritable deficiency in coagulation factor IX (FIX) activity. The severity of the HB bleeding phenotype is dependent on the residual FIX activity: people with severe disease (FIX activity $<1 \%$ of normal) have frequent spontaneous bleeds; people with moderate disease (FIX activity $1 \%-5 \%$ of normal) rarely have spontaneous bleeds but bleed with minor trauma; and people with mild disease (FIX activity 5\%-40\% normal) bleed only during invasive procedures or trauma (1). Modest increases in FIX activity, therefore, have an important clinical effect, making HB highly amenable to treatments, including gene therapy.

The first in-human liver-directed gene therapy clinical trial with an adeno-associated virus (AAV) vector was carried out in subjects with HB (2). The vector contained a FIX-WT transgene, and shortterm efficacy was observed with FIX activity levels up to $12 \%$ normal (2). This approach was limited by a vector dose-dependent cellular immune response targeting AAV capsid proteins presented by transduced hepatocytes $(2,3)$. Immune-mediated destruction of these hepatocytes, clinically manifested by an asymptomatic increase in plasma liver enzyme levels, led to a loss of circulating FIX protein $(2,3)$. A subsequent study demonstrated that the rapid initiation of steroid immunosuppression could reduce the loss of FIX activity, and sustained FIX activity levels of about $5 \%$ normal were observed in the high-dose cohort $(4,5)$. Steroids have been effective in reducing the loss of transgene expression in most, but not all, AAV liver-directed gene therapy trials (reviewed in ref. 6).

Hepatotoxicity, as defined by elevated liver enzymes, has been observed in at least some subjects in all AAV gene transfer trials for $\mathrm{HB}(2,4-7)$ as well as for hemophilia A (HA; ref. 8). However, the lack of identifiable anti-AAV capsid immune cells in some studies suggests that several biological mechanisms may contribute (7-9). Moreover, the AAV-related cellular immunogenicity has not been fully reproduced in preclinical models. As such, lowering the therapeutic vector dose is the most reliable strategy to avoid 
these AAV-related hepatotoxicities, including the use of transgenes with advantageous properties (6). To this end, the high specific activity FIX variant R338L (FIX-R338L; Padua; ref. 10) has recently emerged as the standard transgene for HB gene therapy.

Preclinical studies in small- and large-animal models following AAV liver- or muscle-directed gene transfer demonstrated long-term expression of FIX-R338L without increased immunogenicity compared with FIX-WT (11-16). Based on these preclinical studies, we recently showed that AAV gene therapy with FIX$\mathrm{R} 338 \mathrm{~L}$ in adult $\mathrm{HB}$ subjects allowed for a 4-fold lowering of the therapeutic vector dose but a 6-fold increase in the sustained FIX activity levels, compared with earlier studies using FIX-WT $(4,5,10)$. Importantly, this 4-fold lowering of the vector dose was also associated with a decrease in the number of subjects with hepatotoxicity from 4 of $6(67 \%$; refs. 4,5$)$ to 2 of $10(20 \%$; ref. 10$)$. This success has prompted the incorporation of FIX-R338L into other HB gene therapy programs that previously relied on FIX-WT transgenes.

It is concerning, however, that higher FIX activity levels are being targeted and larger subject cohorts are being recruited without a clear understanding of the biochemical mechanism of FIX-R338L hyperactivity. The recent reports of unanticipated thrombotic complications in several novel therapies for hemophilia, designed to promote hemostasis by avoiding regulatory mechanisms (17-19), emphasize the importance of comprehensively defining the procoagulant activity of new treatment strategies for hemophilia. To address these questions, we instituted a study using purified recombinant (r) protein of FIX variants to evaluate differences in activation, inactivation, and activity in both purified-protein and plasma-based systems. Using a newly developed experimental system where the role of activated factor VIII (FVIIIa) cofactor activity is replaced by the unique reagent emicizumab $(17,20-23)$, we show that the high specific activity of FIX-R338L is dependent on an optimized interaction with its cofactor, FVIIIa, and is not due to differences in FIX activation or inactivation. We also observed the same dependence on FVIII/FVIIIa cofactor activity with transgene-expressed FIX-R338L from HB gene therapy subjects. Combined, these findings suggest that molecular regulation of FIX-R338L is similar to that of FIX-WT. These results justify the continued use of FIX-R338L in AAV-based gene therapy studies for HB by helping alleviate the safety concern of unpredictable thrombogenicity. Moreover, they provide new insights into the underlying biochemistry of FIX activity.

\section{Results}

Activated FIX (FIXa) complexes with its cofactor, FVIIIa, to form the intrinsic Xase enzyme complex (FIXa-FVIIIa), which is responsible for the proteolytic activation of coagulation factor X (FX) through the intrinsic pathway during sustained coagulation $(24,25)$. The proteolytic activity of FIXa toward FX increases at least $10^{5}$-fold upon FVIIIa cofactor binding (26). FIX is activated by either activated factor XI (FXIa) or activated factor VII/tissue factor (FVIIa/TF) and inactivated largely by antithrombin (AT; refs. 24, 27, 28). To identify the molecular mechanism of the hyperactivity of FIX-R338L, we compared all of these reactions with rFIX/FIXa-WT and rFIX/FIXa-R338L in both plasma-based assays and purified-protein systems.

Clotting activity of $r F I X-W T$ and $r F I X-R 338 L$. The Padua substitution R338L was introduced into human FIX-WT through site-directed mutagenesis. Identical chromatography procedures were used to purify rFIX-WT and rFIX-R338L to homogeneity from conditioned media from stably transfected HEK 293 clones. The high purity of rFIX-WT and rFIX-R338L is shown in Supplemental Figure 1; supplemental material available online with this article; https://doi.org/10.1172/jci.insight.128683DS1. The hyperactivity of rFIX-R338L relative to rFIX-WT was confirmed by testing the ability of each protein to restore fibrin clot formation to HB plasma with an assay based on activated partial thromboplastin time (aPTT) (Figure 1). In this experiment, rFIX-R338L protein decreased the time for fibrin clot formation substantially more than similar amounts of rFIX-WT protein. Moreover, the rFIX-R338L curve was left shifted compared with the rFIX-WT curve such that substantially less rFIX-R338L than rFIX-WT was needed for similar clot times. The determined specific activity of rFIX-R338L and rFIX-WT from this assay was $1900 \pm 350$ $\mathrm{U} / \mathrm{mg}$ and $250 \pm 50 \mathrm{U} / \mathrm{mg}$, respectively, consistent with the activity of commercial rFIX-WT HB products (29) and the 8-fold higher activity of FIX-R338L compared with FIX-WT observed in the affected Padua family members (30), animal models (11-16), and gene therapy subjects (10).

Activation of FIX-R338L and FIX-WT by FXIa is similar. Though in vivo FIX is activated by FXIa and FVIIa/TF, the former is the exclusive pathway during the aPTT-based FIX/FIXa specific activity measurements. To assess the role of activation in the high specific activity of FIX-R338L, we compared the rate of activation of FIX-338L and FIX-WT with catalytic amounts of FXIa (30 pM; Figure 2, A and B). At therapeutically relative concentrations of FIX (from $110 \%$ to $14 \%$ normal), we observed that the rates of 


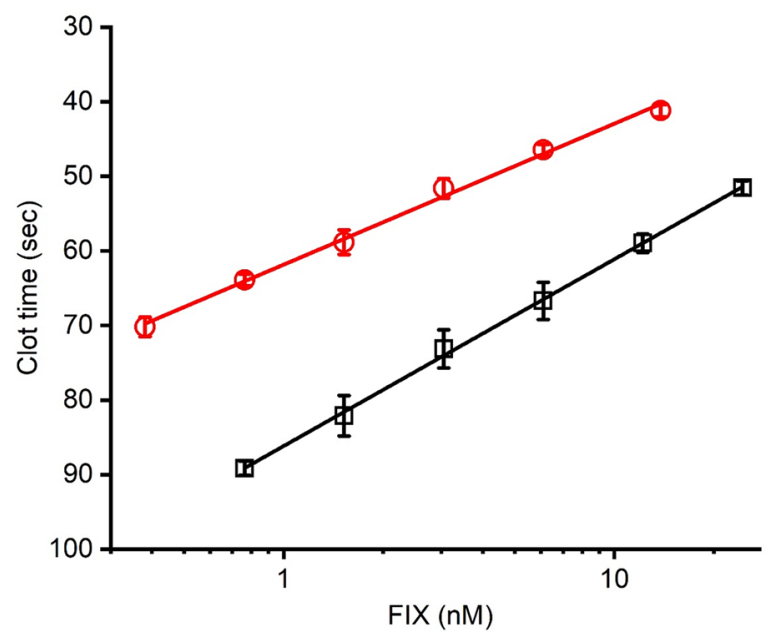

Figure 1. Fibrin clot formation by FIX-WT and FIX-R338L in HB plasma. Dilutions of $r F I X-W T$ and $r F I X-R 338 L$ were added to HB plasma and mixed with an aPTT reagent. After incubation for 180 seconds, clotting was initiated with the addition of $\mathrm{CaCl}_{2}{ }^{-}$. Points represent the mean $(n=2)$ and error bars represent $\pm \mathrm{SD}$. Solid line is a linear fitting $\left(R^{2} \geq 0.99\right.$ for all plots). Data is representative of 4 experiments.

FIX-R338L and FIX-WT activation are similar (Figure 2A). Likewise, the enzyme kinetics of FXIa toward FIX-R338L and FIX-WT are similar (Figure 2B). These data indicate that differences in activation by FXIa are not responsible for the 8-fold hyperactivity of FIX-R338L compared with FIX-WT.

Inactivation of FIXa-R338L and FIXa-WT by AT is similar. FIXa is inactivated by AT, which acts as a suicide substrate by irreversibly binding to the active site of FIXa in the protease domain. To determine whether resistance to AT contributes to the high specific activity of FIXa-R338L, we determined the rate of AT-induced inhibition of rFIXa-R338L and rFIXa-WT (Figure 2C). We observed that at physiologically relevant AT concentrations $(2 \mu \mathrm{M})$, rFIXa-R338L and rFIX-WT were inhibited at comparable rates (Table 1). This result suggests that FIXa-R338L and FIXa-WT are inactivated similarly by AT.

FIXa-R338L has increased rate of FX activation compared with FIXa-WT only in the presence of cofactor FVIIIa. We also compared the proteolytic activity of rFIXa-R338L to rFIXa-WT. For a peptidyl substrate, rFIXaR338L and rFIXa-WT displayed similar activities (Figure 3A and Table 1). Likewise, in the absence of the cofactor VIIIa, rFIXa-R338L and rFIXa-WT had similar enzyme kinetics for the proteolytic activation of the physiological substrate FX (Figure 3B and Table 1). These results are consistent with the observation that rFIXa-R338L and rFIXa-WT had similar activity for the suicide substrate AT (Figure 2C). Combined, rFIXa-R338L and rFIXa-WT have comparable activity for 3 distinct substrates in the absence of FVIIIa cofactor. These data indicate that the active site of rFIXa-R338L is similar to rFIXa-WT.

However, we observed that FIXa-R338L and FIXa-WT have distinct enzyme kinetics for FX activation in the presence of FVIIIa (Figure 3C and Table 1). In Figure 3C, the Michaelis-Menten parameters $\left(K_{m}\right.$ and $\left.k_{C A T}\right)$ of the FIXa-FVIIIa complex are both about 3-fold higher for FIXa-R338L than FIXa-WT. The catalytic efficiency $\left(k_{C A T} / K_{m}\right)$ of the FIXa-R338L and FIXa-WT intrinsic Xase complex are comparable (both $29 \mu \mathrm{M}^{-1} \mathrm{~s}^{-1}$ ). However, the rate of FXa generation at physiological FX concentrations is predicted by these parameters to be 2-fold higher for the FIXa-R338L complex than the FIXa-WT complex because the $K_{m}$ for both complexes remains less than the plasma FX concentration ( $180 \mathrm{nM})$. Thus, at physiologically relevant concentrations, FIXa-R338L bound to FVIIIa is expected to exhibit enhanced FXa generation compared with FIXa-WT bound to FVIIIa.

The role of the FVIIIa binding affinity was assessed by measuring the FXa generation by the FIXa-FVIIIa complex, which is $10^{5}$-fold faster than FXa generation by FIXa without FVIIIa (Table 1). As seen in Figure 3D, the $a p p K_{D}$ values of FIXa-R338L and FIXa-WT for FVIIIa in this assay were comparable (Table 1), which suggests that FVIIIa binds rFIXa-WT and rFIX-R338L similarly. These app $K_{D}$ values were used to determine the amount of intrinsic Xase complex in Figure 3C. These data imply that the R338L substitution does not substantially change the affinity between FIXa and FVIIIa; rather FIXa-R338L bound to FVIIIa is more efficient at converting FX to FXa compared with FIXa-WT bound to FVIIIa. This enhancement of FXa generation by FIXa-R338L bound to FVIIIa compared with FIXa-WT bound to FVIIIa suggests the R338L substitution improves the allosteric activation of FIXa by FVIIIa. As predicated by the relative enzyme kinetic 
A

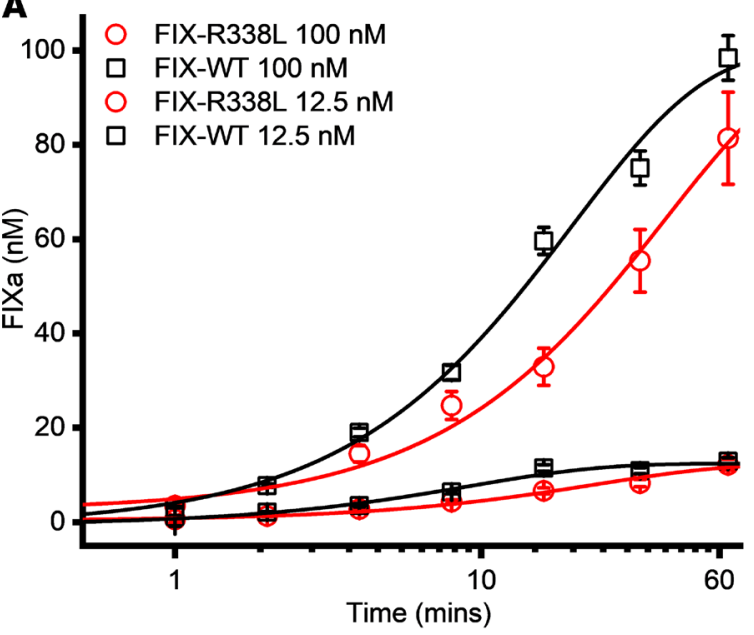

B

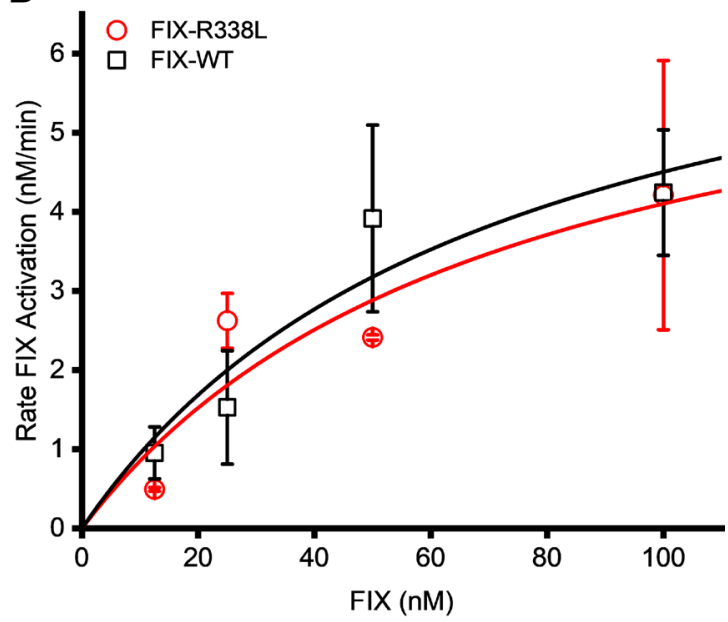

C

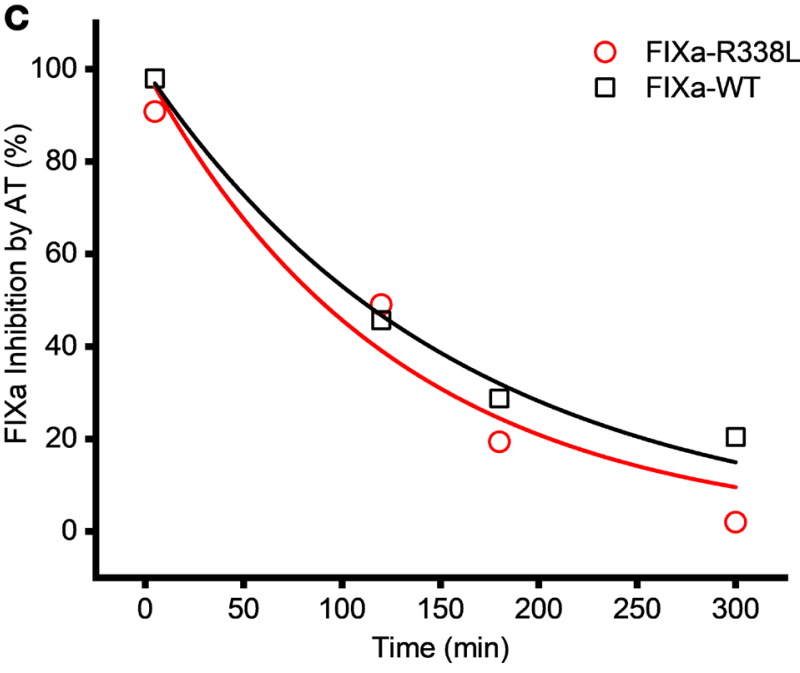

Figure 2. Activation and inactivation of rFIX/FIXa-WT and rFIX/FIXa-R338L. (A) The rate of activation of FIX-WT and FIX-R338L by FXIa (30 pM) was determined by Western blotting. The amount of FIXa generated as a function of time starting with 100 or $12.5 \mathrm{nM} \mathrm{FIX}$ as indicated. The $x$ axis scale is logarithmic for clarity. Each point is the determined [FIXa], and the error bars represent the standard error of the proportionality constants as detailed in Methods. Solid lines are exponential fittings. (B) Enzyme kinetics of FXla activation of FIX-WT and FIX-R338L. Each data point represents the mean of the determined rate of FIXa formation and FIX decay, and the error bars show \pm SD. Solid lines are Michaelis-Menten equation fittings. (C) Inactivation of FIXa-WT and FIXa-R338L by AT. FIXa-WT and FIXa-R338L protein ( $500 \mathrm{nM})$ was incubated with and without $2 \mu \mathrm{M}$ AT. Pseudo-first order rate constants of FIXa-WT and FIXa-R338L inhibition by AT were determined by single-exponential fitting $\left(R^{2}>0.95\right)$ of residual FIXa activity.

parameters of FIXa-R338L and FIXa-WT, the specific activity ratio of FIX-R338L to FIX-WT increases with increasing FX concentration in plasma-based clotting assays (Supplemental Figure 2).

Hyperactivity of FIX/FIXa-R338L in plasma assays requires FVIIIa cofactor. To determine whether this increase in FX activation by the FIXa-R338L-FVIIIa complex relative to the FIXa-WT-FVIIIa complex observed in purified-protein assays (Figure 3 ) is sufficient to produce the 8-fold increased specific activity of FIX-R338L compared with FIX-WT observed in clotting assays (Figure 1), we developed a new plasma-based assay that can measure FIX/FIXa activity without FVIII/FVIIIa. Unlike in the purified-protein assays, FVIII/FVIIIa activity is required to measure FIX/FIXa activity in plasma because the absence of FVIII/FVIIIa activity in plasma causes HA.

In these experiments, endogenous FVIII/FVIIIa cofactor activity in HB plasma is replaced by emicizumab. Emicizumab is a bispecific antibody that efficiently brings together FIX/FIXa and FX/FXa, forming an intrinsic Xase complex without FVIIIa (refs. 20-22 and Supplemental Figure 3). It is efficacious for the prevention of bleeding for HA patients with and without inhibitors $(17,23)$. To inhibit the endogenous FVIII/FVIIIa activity in plasma, we tested a series of monoclonal, neutralizing anti-FVIII antibodies to attain a combination that can sufficiently inhibit FVIII/FVIIIa activity in plasma to less than or equal to 
Table 1. Comparison of biochemical parameters of rFIXa-WT and rFIXa-R338L

\begin{tabular}{|c|c|c|c|c|c|c|c|c|c|c|}
\hline & \multicolumn{4}{|c|}{ No cofactor } & \multicolumn{3}{|c|}{ FVIIIa } & \multicolumn{3}{|c|}{ Emicizumab } \\
\hline & $\begin{array}{c}\text { AT inhibition } \\
\left(\mathrm{min}^{-1}\right)\end{array}$ & $\begin{array}{c}\text { Peptidyl } \\
\text { activity }\left(\mu \mathrm{M}^{-1} \mathrm{~s}^{-1}\right)\end{array}$ & $K_{m}(\mathrm{nM})$ & $k_{C A T}\left(\mathrm{~s}^{-1}\right)$ & $K_{m}(n M)$ & $k_{C A T}\left(s^{-1}\right)$ & $a p p K_{D}(\mathrm{nM})$ & $K_{m}(\mathrm{nM})$ & $k_{\text {CAT }}\left(\mathrm{s}^{-1}\right)$ & $a p p K_{D}(\mathrm{nM})$ \\
\hline FIXa-WT & $\begin{array}{l}0.006 \pm \\
0.0004\end{array}$ & $2.0 \pm 0.1$ & 400 & $8 \pm 1 \times 10^{-5}$ & $41 \pm 6$ & $1.2 \pm 0.1$ & $2.7 \pm 0.3$ & $8 \pm 3$ & $4.4 \pm 0.01 \times 10^{-3}$ & $3.8 \pm 1.6$ \\
\hline FIXa-R338L & $0.008 \pm 0.001$ & $1.7 \pm 0.1$ & 500 & $8 \pm 2 \times 10^{-5}$ & $118 \pm 5$ & $3.4 \pm 0.1$ & $3.5 \pm 0.5$ & $5 \pm 2$ & $6.1 \pm 0.01 \times 10^{-3}$ & $2.7 \pm 0.6$ \\
\hline
\end{tabular}

Parameters and error are determined from the curve fittings in Figures 2 And 3 and Supplemental Figure 8.

$1 \%$ normal (Supplemental Figure 4). These antibodies and emicizumab were added to HB plasma, and a standard curve using commercial rFIX-WT was determined to relate clotting time to FIX activity for emicizumab-conditioned HB plasma.

In unmodified HB plasma, we observed that FIX-R338L had the expected 8-fold increased specific activity compared with FIX-WT as measured by an aPTT-based clotting assay (Figure 4A). However, when the endogenous FVIII/FVIIIa was replaced by emicizumab, zymogen rFIX-WT had a slightly higher specific activity than rFIX-R338L (Figure 4A). The FVIIIa-dependent activity was estimated as the ratio of the specific activities without and with emicizumab. This ratio was 0.8 and 9.3, respectively, for rFIX-WT and rFIX-R338L, indicating that the 8-fold hyperactivity of rFIX-R338L was FVIIIa dependent.

Similar to these results between the zymogen forms of the FIX variants, we also observed that the increased specific activity of rFIXa-R338L compared with rFIXa-WT was abrogated by the replacement of FVIIIa cofactor activity with emicizumab (Figure 4B). Combined, these results indicate that the observed hyperactivity of FIX/FIXa-R338L measured in clotting assays was wholly dependent on its enhanced interaction with FVIII/FVIIIa. As was observed in the purified-protein systems (Table 1), FVIII/FVIIIa cofactor activity was required for FIX/FIXa-R338L advantages over FIX/FIXa-WT in clotting assays.

The observed hyperactivity of rFIXa-R338L compared with rFIXa-WT in the clotting assay (Figure 4B) also indicates that the mechanism of increased specific activity is due to differences in the activated form rather than the zymogen form, which is expected as only FIXa, and not FIX, binds FVIIIa (26). This conclusion is consistent with the similar rates of activation measured in Figure 2A of FIX-WT and FIX-R338L. The observed increased specific activity of rFIXa-R338L relative to rFIXa-WT in Figure 4B also controls for the potential of undetectable amounts of rFIXa-R338L contaminating the rFIX-R338L samples and increasing the apparent specific activity.

We also determined the dependence of FVIIIa cofactor activity on the enhanced ability of FIX/FIXaR338L compared with FIX/FIXa-WT to restore thrombin production in HB plasma in the thrombin generation assay (TGA). This assay provides a more global perspective on coagulation because it evaluates procoagulant activity over 90 minutes compared with the few minutes until fibrin clot formation in the clotting assay. In unmodified HB plasma, both FIX variants restored thrombin generation in a dose-dependent manner (Figure 5, A and B), but substantially less rFIX-R338L than rFIX-WT was required as is evidenced by the left-shifted curves of the TGA parameters of FIX-R338L compared with FIX-WT (Figure 5D and Supplemental Figure 5). The estimated $\mathrm{EC}_{50}$ of these parameters for FIX-WT is 3- to 10-fold higher than for FIX-R338L (Table 2), consistent with FIX-R338L having an increased procoagulant activity compared with FIX-WT. However, when emicizumab replaces the endogenous FVIII/FVIIIa, FIX-R338L and FIX-WT exhibit similar thrombograms (Figure 5C) and TGA parameters (Figure 5D, Supplemental Figure 5, and Table 2). These results indicate that the enhanced thrombin generation of FIX-R338L over FIX-WT is dependent on FVIII/FVIIIa activity.

We also determined the ability of the activated protease forms of the FIX variants to restore thrombin generation in HB plasma (Supplemental Figure 6 and Table 2). Similar to results with the zymogen forms, the increased thrombin generation of rFIXa-R338L compared with rFIXa-WT was ablated when emicizumab replaced the endogenous FVIII/FVIIIa. These results further support our model that the enhanced thrombin generation of FIXa-R338L over FIXa-WT is mainly dependent on FVIII/FVIIIa activity. They also control for the possibility that the hyperactivity of FIX-R338L over FIX-WT observed in the TGA (Figure 5) is due to trace amounts of activated protease.

Emicizumab binds both FIX/FIXa and FX/FXa in their EGF domains (22). As such, it seems very unlikely that the results depicted in Figures 4 And 5 could be due to differences in emicizumab binding to 
A

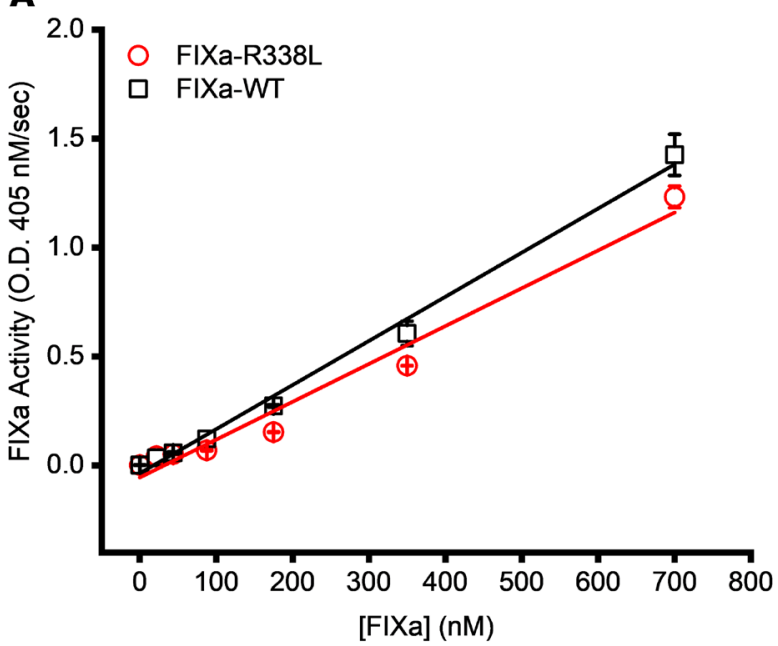

C

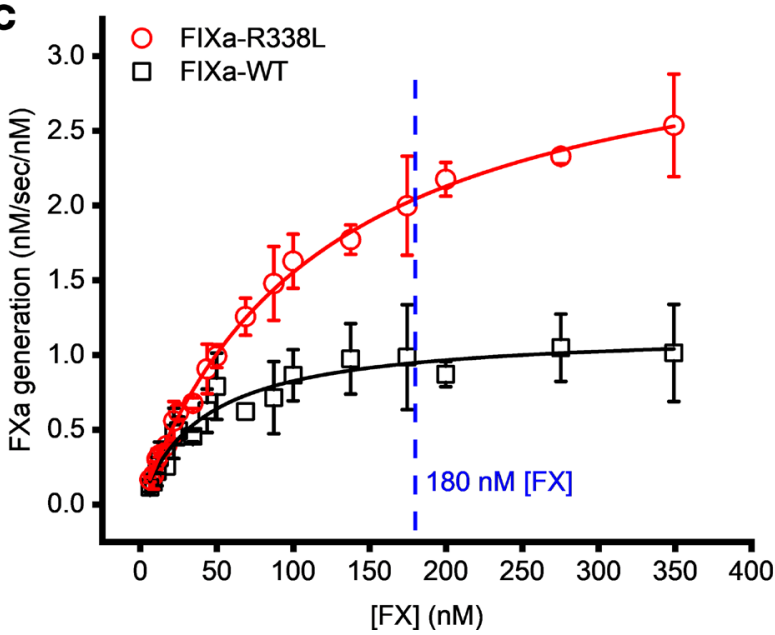

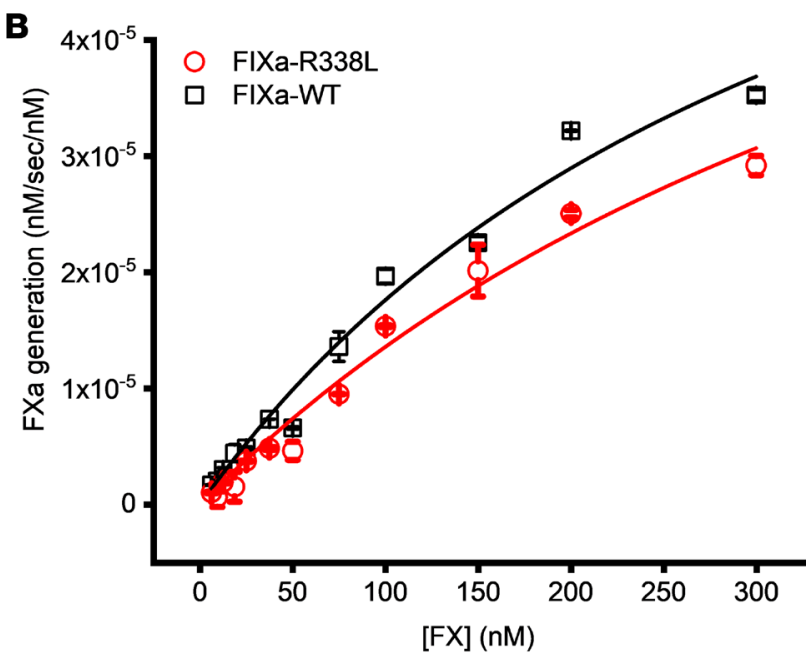

D

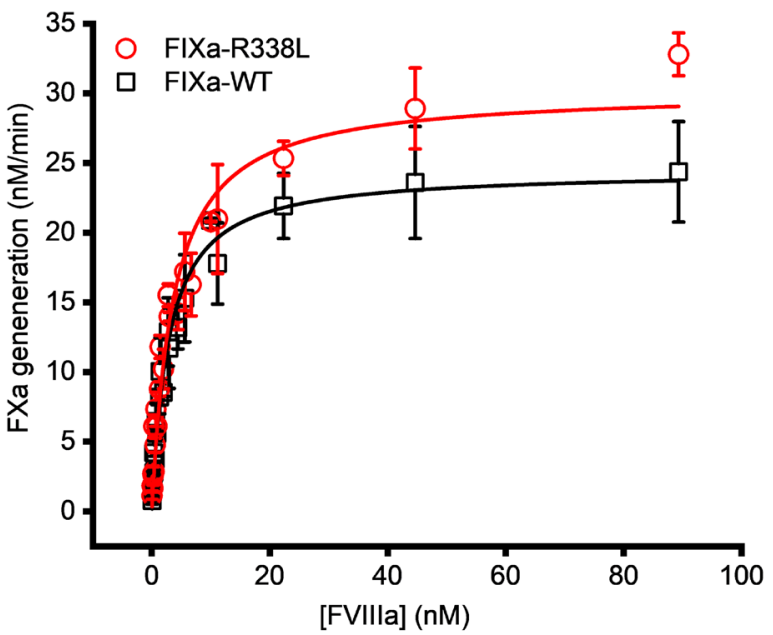

Figure 3. Activity of rFIXa-WT and rFIXa-R338L with and without FVIIla. (A) Activity of FIXa-WT and FIXa-R338L for peptidyl substrate. Points represent the mean $(n=2)$ and error bars show \pm SD. Linear fitting $\left(R^{2} \geq 0.97\right)$ yields biomolecular rate constant. (B) Enzyme kinetics of the activation of FX by FIXa without FVIIla cofactor. Points represent the mean $(n=3)$ of FXa activity and error bars show \pm SD. Solid lines are Michaelis-Menten fittings $\left(R^{2} \geq 0.97\right)$. (C) Enzyme kinetics of the activation of FX by $0.3 \mathrm{nM}$ FIXa with $1.8 \mathrm{nM}$ FVIIla. Points represent the mean $(n=2-3)$ and error bars show \pm SD. Data are representative of 3 experiments. Solid lines are Michaelis-Menten fittings $\left(R^{2} \geq 0.94\right)$. (D) FVIIla binding to $0.3 \mathrm{nM}$ FIXa assayed by FIXa-FVIIla complex FXa generation; initial [FX] is $300 \mathrm{nM}$. Points represent the mean $(n=2-3)$ and error bars \pm SD. Data are representative of 3 experiments. Solid lines are fittings of a quadratic binding equation $\left(R^{2} \geq 0.96\right)$.

FIX/FIXa-R338L and FIX/FIXa-WT because the R338L substitution resides in the protease domain of FIX (Supplemental Figure 7). In support of this, we found that FIXa-R338L and FIXa-WT had comparable $a p p K_{D}$ with emicizumab (Supplemental Figure 8A and Table 1), indicating that emicizumab binds similarly to FIXa-R338L and FIXa-WT. We also measured the enzyme kinetics parameters of FIXa-R338L and FIXa-WT with emicizumab (Supplemental Figure 8B and Table 1), which are consistent with previously reported values (21). The measured $a p p K_{D}$ of emicizumab with FIXa-WT in this functional assay with phospholipids is higher than the $K_{D}$ determined using immobilized, monospecific, 2-armed antibodies (22). However, an analogous discrepancy is also noted in the published $K_{m}$ and $K_{D}$ values $(21,22)$, suggesting that these differences are likely assay dependent. Combined, our results indicate that the intrinsic Xase complex with emicizumab (FIXa-emicizumab) are similar for FIXa-WT and FIXa-R338L. These data further support our model that the hyperactivity of FIX/FIXa-R338L requires FVIIIa cofactor activity.

Hyperactivity of FIX-R338L expressed after AAV liver-directed gene therapy in HB subjects requires FVIIIa cofactor. To validate the clinical relevance of our in vitro observations that the hyperactivity of rFIX/FIXaR338L requires FVIIIa cofactor activity, we determined the FIX activity in plasma samples from subjects 
A

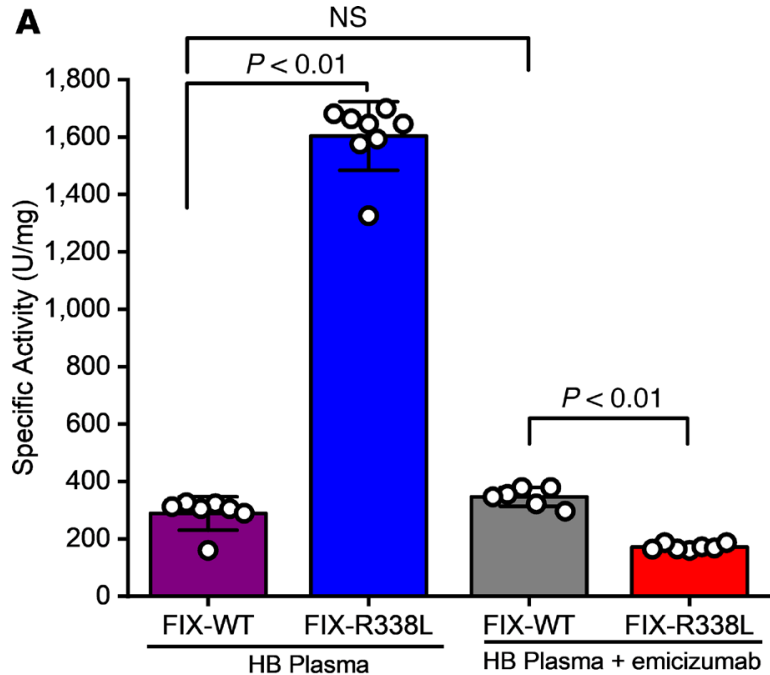

B

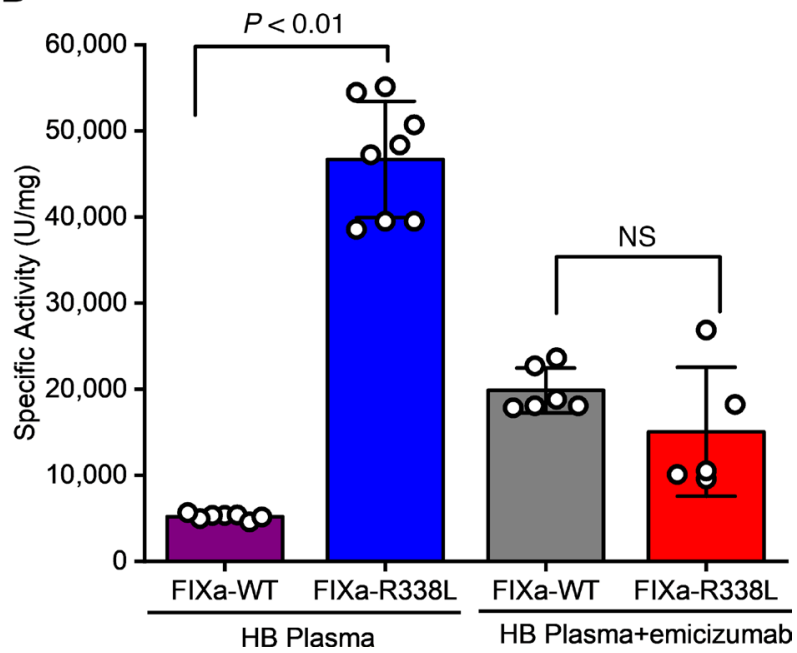

Figure 4. Comparison of specific activity of FIX/FIXa-WT and FIX/FIXa-R338L in HB plasma and HB plasma with emicizumab. The clotting activity of each variant, either as zymogen (A) or as activated protease (B), was measured with an aPTT-based clotting assay. Emicizumab was added to a final plasma concentration $100 \mathrm{nM}$. Each circle is a distinct clotting time measurement $(n=5-8)$, bars represent mean of data, and error bars show \pm SD. Means were compared with a 2-tailed $t$ test with $P$ values $\geq 0.05$ considered not significant (NS).

with HB treated at the Children's Hospital of Philadelphia with AAV-FIX-R338L (SPK-9001) gene therapy (10). After receiving AAV gene therapy, all these subjects persistently express FIX-R338L, which has resulted in a near phenotypic cure in most participants (10). Four samples from 3 subjects were available with FIX activities that ranged from $17 \%$ to $53 \%$ normal FIX activity after gene therapy. In unmodified HB plasma, FIX activities were similar (within $0.04 \mathrm{IU} / \mathrm{mL}$ ) to the activities determined in the clinical laboratory. However, when the endogenous FVIIIa cofactor activity of the HB plasma was replaced by emicizumab, the FIX activity was reduced about 8-fold (Figure 6A); this decrease is consistent with our observations that the hyperactivity of rFIX/FIXa-R338L requires FVIIIa cofactor activity (Figures 4 And 5).

Just as we determined in Figure 4A, the FVIIIa-dependent hyperactivity of FIX-R338L expressed after gene therapy can be estimated from the ratio between the FIX activity measured without and with emicizumab (Figure 6B), while accounting for the subject's endogenous pre-gene therapy FIX activity. Samples 1 to 3 are from subjects with severe HB before gene therapy (endogenous FIX activity < $1 \%$ normal) while sample 4 is from a subject with moderate HB (endogenous FIX activity about $2 \%$ normal). The determined FVIIIa-dependent hyperactivity of transgene-expressed FIX-R338L from these samples is between 7 and 10, comparable with our results using rFIX-R338L. These results indicate that the hyperactivity of FIX-R338L expressed in subjects with HB after AAV gene therapy was dependent on FVIIIa cofactor activity, just as we observed for the hyperactivity of rFIX/FIXa-R338L. This similarity further substantiates our model that the increased specific activity of rFIX-R338L is mediated through an enhancement of FVIIIa-induced allosteric activation of FIXa. Thus, transgene-expressed FIX-R338L is likely regulated similarly to endogenous FIX-WT.

\section{Discussion}

The therapeutic armamentarium for hemophilia is rapidly expanding $(18,19,31)$. However, the clinical development of several new products has been marred by unanticipated thrombotic complications and subject deaths (17-19). The only published clinical gene therapy trial in subjects with HB expressing FIXR338L ( $n=10)$ used a single low vector dose that resulted in most subjects $(n=9)$ having less than $50 \%$ normal FIX activity (mean 35\% normal; ref. 10). Elucidating the mechanism of the increased specific activity of FIX-R338L has important safety implications as gene therapy with FIX-R338L advances into pivotal phase III studies with larger study cohorts as well as higher targeted FIX activity levels.

Potential mechanisms of increased specific activity of FIX include enhancements in FIX activation, resistance to AT inactivation (32), FVIIIa-independent activity (33, 34), and improvements in the interaction with FVIIIa (35-37). Herein, we provide mechanistic data that demonstrate FIX/FIXa-R338L and FIX/ 

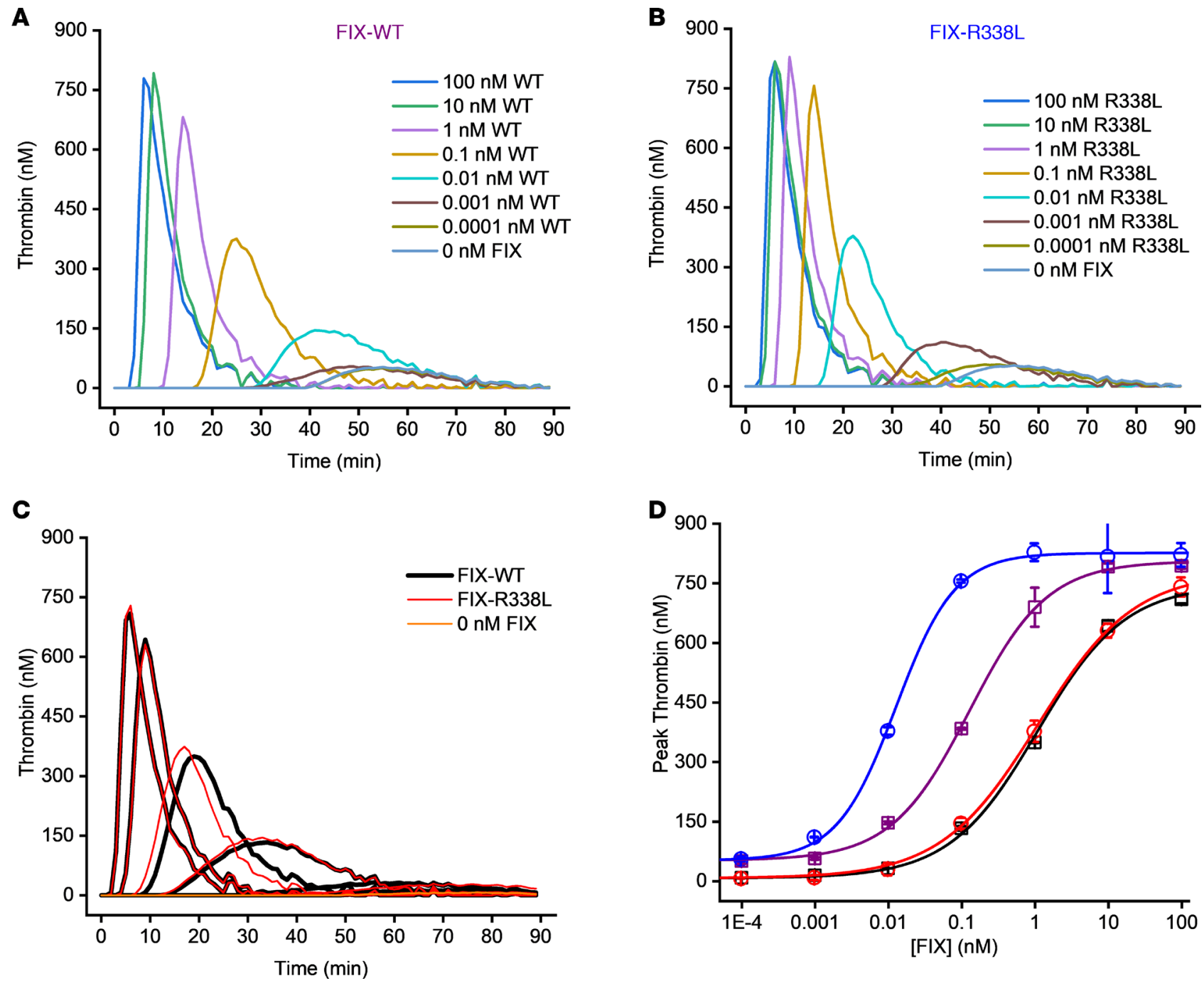

Figure 5. Thrombin generation of zymogen rFIX-WT and rFIX-R338L in HB plasma and HB plasma with emicizumab. rFIX-WT-dependent (A) and rFIX-R338L-dependent (B) thrombin generation in unmodified HB plasma. (C) rFIX-WT-dependent (black) and rFIX-R338L-dependent (red) thrombin generation in $\mathrm{HB}$ plasma conditioned with $100 \mathrm{nM}$ emicizumab and anti-FVIII antibodies. The same FIXa protein samples were used for experiments in HB plasma (A and B) and HB plasma with emicizumab (C). Thrombograms are the mean of $n=2$ measurements. (D) Peak thrombin as a function of added rFIX protein. Points represent the mean $(n=2)$ and error bars show \pm SD. Data points were empirically fitted to a logistic plot (solid lines) to estimate the half maximal effective concentration $\left(\mathrm{EC}_{50}\right)\left(R^{2} \geq 0.98\right.$ for all plots). The analogous plots of ETP and lag time are in Supplemental Figure 5. Data are representative of 2 to 4 experiments.

FIXa-WT are regulated similarly at a molecular level and that the hyperactivity of FIX/FIXa-R338L is the result of an enhanced interaction with FVIIIa that causes an increased rate of FX activation in physiological conditions. Without FVIIIa, both rFIXa-WT and rFIXa-R338L have similar activity for both peptidyl substrates and FX. However, when complexed with FVIIIa, rFIXa-R338L demonstrates several-fold increased FXa generation at physiological FX concentrations compared with rFIXa-WT, even though FVIIIa binding affinity to FIXa-WT and FIXa-R338L is comparable. Rather, the allosteric activation of FIXa by FVIIIa for FXa generation is enhanced in FIXa-R338L, compared with FIXa-WT. The substantial enhancement of rFIX/FIXa-R338L compared with rFIX/FIXa-WT in plasma-based assays is completely dependent on its FVIIIa cofactor interaction. The hyperactivity of rFIX/FIXa-R338L is entirely ablated in both a clotting assay and a TGA when FVIIIa is replaced by emicizumab. Emicizumab is a bispecific antibody designed to be an "FVIII mimetic" that was recently approved for HA patients with and without inhibitors (17, 20-23). As a reagent, it provides a unique opportunity to test the role of FVIIIa cofactor activity in plasma.

The reported specific activity of human and canine FIX-R338L ranges between 5- and 15-fold higher than the FIX-WT ortholog, depending on the reagents and source of protein, with about 8-fold being 
Table 2. Comparison of the $\mathrm{EC}_{50}$ of thrombin generation parameters of FIX/FIXa-R338L and FIX/FIXa-WT in HB plasma

\begin{tabular}{|c|c|c|c|c|c|c|}
\hline & FIX/FIXa-R338L & FIX/FIXa-WT & Ratio WT/R338L & $\begin{array}{c}\text { FIX/FIXa-R338L + } \\
\text { emicizumab }\end{array}$ & $\begin{array}{c}\text { FIX/FIXa-WT + } \\
\text { emicizumab }\end{array}$ & $\begin{array}{c}\text { Ratio WT/R338L + } \\
\text { emicizumab }\end{array}$ \\
\hline \multicolumn{7}{|c|}{ FIX (nM) } \\
\hline Peak thrombin & $0.013 \pm 0.001$ & $0.13 \pm 0.01$ & 10 & $1.1 \pm 0.2$ & $1.1 \pm 0.1$ & 1 \\
\hline ETP & $0.003 \pm 0.001$ & $0.009 \pm 0.003$ & 3 & $0.05 \pm 0.007$ & $0.06 \pm 0.003$ & 1 \\
\hline \multicolumn{7}{|c|}{ FIXa (pM) } \\
\hline Peak thrombin & $4.6 \pm 0.5$ & $30 \pm 10$ & 7 & $200 \pm 100$ & $200 \pm 70$ & 1 \\
\hline
\end{tabular}

the most consistently observed (reviewed in ref. 6), which is in agreement with our clotting assay results. However, the procoagulant advantages of FIX/FIXa-R338L over FIX/FIXa-WT that we observed were assay dependent, with the clotting assay specific activity being about 8-fold while the determined $\mathrm{EC}_{50}$ of the TGA parameters ranged from 3- to 10-fold. Indeed, the fold difference in thrombin generation between FIX/FIXa-R338L and FIX/FIXa-WT was highly dependent on the FIX/FIXa protein concentration used. We also observed that the hyperactivity of FIX/FIXa-R338L over FIX/FIXa-WT was larger in the plasma-based assays than in the purified-protein system assays, which was likely due to the hyperactivity being amplified down the coagulation cascade in the former experiments (38). A similar phenomenon of higher plasma-based activities compared with purified system FXa generation assays has been noted in TF-independent, hyperactive FVIIa variants (39) and other high-specific activity FIX/FIXa variants (40). We speculate that this difference likely contributes to the observed 2-fold discrepancies in the 1-stage clotting and chromogenic FIX activity assays observed in HB gene therapy subjects expressing FIX-R338L (10).

We also observed that the high specific activity of transgene-expressed FIX-R338L in subjects with HB after AAV gene therapy was also dependent on FVIIIa cofactor activity, consistent with our data using rFIX-R338L protein. Discrepancies in the measured FVIII activity between rFVIII and transgene-expressed FVIII have recently been appreciated (reviewed in ref. 6). However, the similarity between rFIX-R338L and the transgene-expressed FIX-R338L substantiate the clinical relevance of our results. These results buttress the use of FIX-R338L as a therapeutic for HB and inform on the biochemistry of FIX activity.
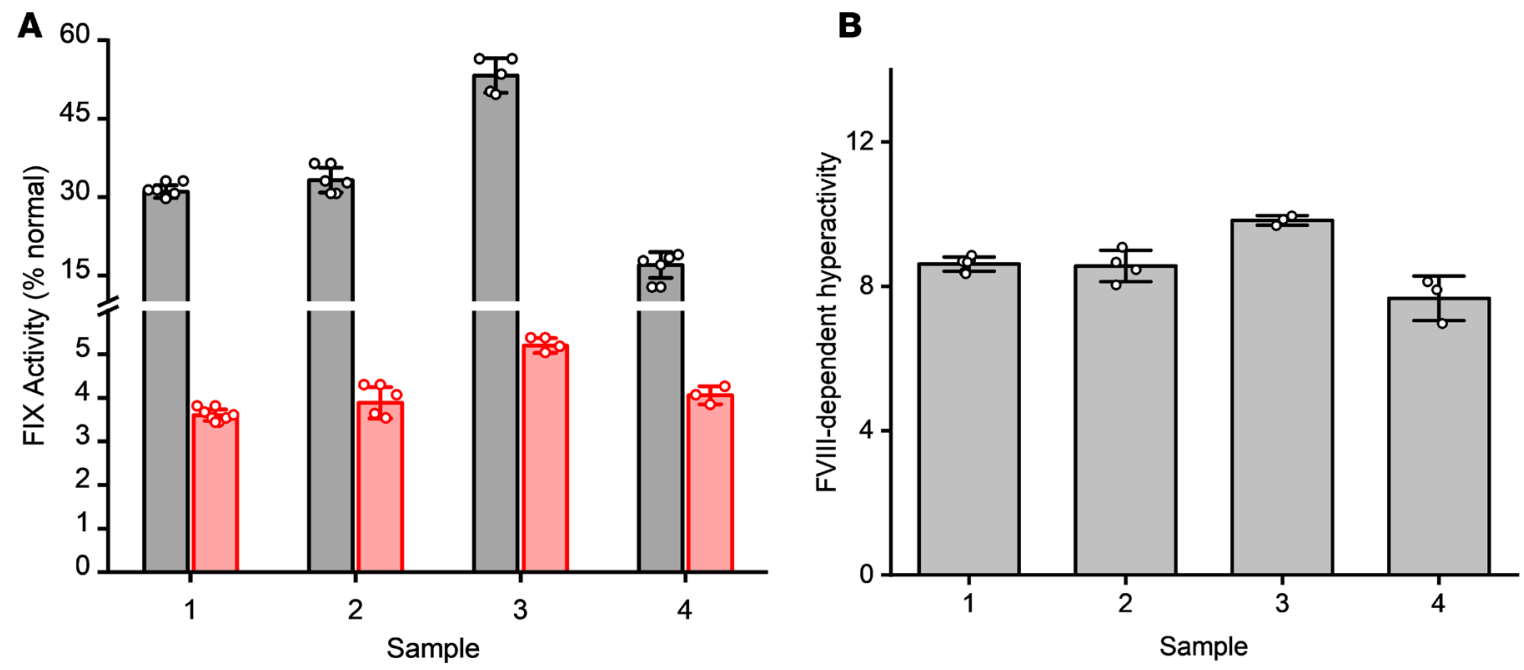

Figure 6. FIX activity of subjects with HB expressing FIX-R338L after AAV gene therapy with HB plasma with and without emicizumab. (A) The FIX activity of FIX-R338L expressed from HB subjects after AAV gene therapy was measured using an aPTT-based clotting assay either in unmodified HB plasma (gray) or HB plasma with $100 \mathrm{nM}$ emicizumab and anti-FVIII antibodies (red). Each circle is a distinct clotting time measurement ( $n=3-6)$, bars represent mean of data, and error bars show \pm SD. (B) The FVIIla-dependent hyperactivity of transgene-expressed FIX-R338L was determined by the ratio between the FIX activity measured without and with emicizumab while accounting for the subject's endogenous FIX activity (before gene therapy). Bars represent mean of data of paired measurements $(n=3-6)$ and error bars show \pm SD. 
Translational implications. This mechanistic basis for the enhanced plasma activity of FIX-R338L described herein complements our previous in vivo safety assessments, and combined, provides a strong rationale that its expanding use in HB gene therapy will be safe. FIX-R338L and FIX-WT in physiological conditions are likely regulated similarly with comparable rates of activation of FXIa and inactivation by AT and the same FVIIIa cofactor requirement. Unknown or alternative regulatory interactions not evaluated $(24,41,42)$ could be different for FIX/FIXa-R338L and FIX/FIXa-WT. However, the striking loss of the hyperactivity of FIX/FIXa-R338L compared with FIX/FIXa-WT in plasma-based assays without FVIII/ FVIIIa activity suggests that the increased specific activity of FIX/FIXa-R338L relative to FIX/FIXa-WT is primarily due to an enhanced allosteric activation of FIXa-R338L relative to FIXa-WT by FVIIIa.

As such, the thrombophilia of FIX-Padua is likely due only to the very elevated FIX activity levels, rather than derangements in the regulation of coagulation. Indeed, only the FIX-Padua proband with FIX activity in excess of $700 \%$ normal developed a thrombosis, while his mother, who was a heterozygote carrier with levels of $330 \%$ normal, never had thrombotic complications, despite multiple pregnancies, which is also a prothrombotic state (30). This conclusion is also supported by our previous observation that in WT mice expressing supratherapeutic FIX activity levels (100\%-2000\% normal), markers of coagulation activation and mortality were the same for mice expressing FIX-R338L or FIX-WT. In other words, the thrombotic risk was due to high FIX activity levels regardless of the FIX variant responsible for the elevated levels, consistent with our biochemical results described here. Combined, our biochemical and preclinical data suggest that the thrombotic risk of FIX-R338L as a therapeutic is probably only dependent on the FIX activity levels and is likely the same as FIX-WT at the identical activity levels. These data support gene therapy and gene editing approaches aimed at achieving higher FIX activity levels than obtained to date.

Biochemical implications. FVIIIa cofactor activity is required for efficient FIXa activation of FX to prevent hemorrhages. Deficiencies in either FVIIIa or FIXa (HA and HB, respectively) result in similar bleeding disorders. At the molecular level, FVIIIa binding to FIXa augments its catalytic site for FX proteolysis through allosteric activation as well as providing additional stabilizing binding interactions for FX. Our results suggest that the enhanced interaction of FVIIIa and FIXa-R338L that is responsible for the increased specific activity of FIX/FIXa-R338L (that is abolished when emicizumab replaces FVIIIa) is likely an improved allosteric activation rather than an increased affinity of FVIIIa for FIXa.

Though described as an "FVIII mimetic," the emicizumab-FIXa enzyme complex actually has distinct enzyme kinetics from the FVIIIa-FIXa complex (21). Although FVIIIa binding results in a 2-fold increase in FX binding and a $10^{5}$-fold increase in the rate of FX activation, emicizumab binding FIXa results in a 20 -fold increase in FX binding and only a $10^{3}$-fold increase in the rate of FX activation (21). The rate of FX activation by the emicizumab-FIXa complex can be further enhanced by the addition of the components of FVIIIa likely to be responsible for this allosteric improvement of the FIXa active site (the FVIII heavy chain or the isolated FVIII-A2 domain; ref. 43). These data indicate that emicizumab can enhance binding of FX to FIXa but has a limited allosteric improvement of the FIXa active site. Our results suggest that the increased specific activity of FIX/FIXa-R338L is dependent on a specific FVIIIa-induced allosteric improvement of the FIXa catalytic site, which is enhanced by the R338L substitution.

Previous loss-of-function studies also support this model of FIX/FIXa-R338L hyperactivity. Mutations in neighboring amino acids (R333, L337) result in FIXa variants with decreased FVIIIa-induced allostery but no measurable change in FVIIIa binding affinity (44). Likewise, loss-of-function mutations in the FVIII-A2 domain can modulate FIXa enzymatic activity but also do not substantially change the FIXa/FVIIIa affinity (45). Thus, our model of FIX/FIXa-R338L hyperactivity is the converse of these previous results where FVIIIa-induced allostery is diminished without substantially changing the FIXa/FVIIIa affinity.

Our results are also consistent with the biochemical characterization of the related variant, FIX/FIXaR338A. FIX-R338A had a 3-fold increased specific activity compared with FIX-WT in an aPTT-based assay, and FIXa-R338A has a 2-fold increase in FX activation compared with FIXa-WT only when complexed with FVIIIa cofactor $(35,36)$. Thus, a similar mechanism of increased activity likely occurs for both FIX-R338L and FIX-R338A. Indeed, the positioning of the $\alpha$-helix containing R338 (Supplemental Figure 7) has been identified as a key contributor to the maturation of the FIXa active site (46).

Evolutionary implications. Curiously, our results also suggest the FIX-WT has not evolved to be an optimal protease of FX activation because the R338L substitution dramatically enhanced its activity. Rather, other evolutionary pressures may have influenced FIX-WT, such as limiting FIX activity to prevent throm- 
bosis. Supporting this conjecture are the earlier observations that (a) R338 is conserved throughout mammalian evolution even though it contains a CpG dinucleotide mutation hotspot and (b) missense mutations are underrepresented at this position in the HB database, with only a single variant (R338P) being associated with disease (47). Combined with our data, these considerations suggest that R338 was specifically conserved to limit FIX activity by diminishing the allosteric interaction between FVIIIa and FIXa. These considerations also imply that there may be additional opportunities to further enhance the potency of FIX-WT, as has already been observed for number of FIX variants (6).

Conclusions. FIX-R338L is being used in multiple phase III gene therapy trials for HB. The mechanistic understanding that the hyperactivity of FIX-R338L is reliant on an enhanced interaction with its cofactor, FVIII, for improved allosteric activation supports both targeting higher FIX activity levels as well as recruiting larger eligible populations of subjects with HB. This specific nature of the gain of function diminishes concerns of dysregulated hemostasis and the potential for thrombotic complications.

\section{Methods}

Expression and purification of zymogen (FIX) and activated (FIXa) FIX-WT and FIX-R338L. Stable FIX-expressing HEK 293 cells (48) were developed using methods previously described $(11,13,36)$. Stable clones were selected from at least 48 single colonies of lipofectamine-transfected cells, and 10-15 $\mu \mathrm{g} / \mathrm{mL}$ vitamin $\mathrm{K}$ was included in the expression media. The rFIX proteins were purified from conditioned media over a series of ion exchange chromatography columns based on FIX activity in a clotting assay. Homogeneity was confirmed with lithium dodecyl sulfate polyacrylamide gel electrophoresis (LDS-PAGE) analysis, as shown in Supplemental Figure 1A. Protein yields were about $0.4 \mathrm{mg} / \mathrm{L}$ of conditioned media. The rFIX concentration was determined by absorbance at $280 \mathrm{~nm}$ immediately before all experiments based on an extinction coefficient of $1.32 \mathrm{mg} / \mathrm{mL} / \mathrm{cm}$.

FIXa was generated by incubating rFIX protein with catalytic amounts ( 1:1000) of FXIa (Haemtech). FIXa was subsequently separated from residual zymogen and FXIa using a Sephacryl 200 gel filtration column (GE Healthcare); homogeneity was confirmed with LDS-PAGE analysis, as shown in Supplemental Figure 1B.

Clotting assays. FIX activity was determined with an aPTT-based 1-stage clotting assay as described previously $(30,49)$. In disposable Stago STart cuvettes, $50 \mu \mathrm{L}$ of plasma from subjects with severe HB with less than $1 \%$ normal FIX activity (George King) was combined with $50 \mu \mathrm{L}$ of analyte and $50 \mu \mathrm{L}$ TriniCLOT silica aPTT reagent (TCoag) and incubated at $37^{\circ} \mathrm{C}$ for 180 seconds in a Stago STart Coagulation Analyzer. Commercial rFIX-WT (Pfizer) was used as a standard to relate clot time to FIX activity.

Kinetics of FIX activation by FXIa. To quantify the enzyme kinetics of FIX activation by FXIa, the conversion of FIX to FIXa was monitored with Western blotting using a commercial FIX/FIXa heavy chain monoclonal antibody, Green Mountain Antibody (GMA-102). In HEPES-buffered saline (HBS), 30 pM FXIa was incubated with 100, 50, 25, or $12.5 \mathrm{nM}$ rFIX protein, FIX-WT, or FIX-R338L. Aliquots were taken over 1 hour and immediately mixed with LDS sample buffer to quench proteolysis. A single blot using anti-mouse IgG DyLight-800 (Rockland) as a secondary antibody was used for all time points for each initial concentration of FIX-WT or FIX-R338L. The fluorescence of each blot was measured using an Odyssey Imaging Systems (LI-COR Biosciences), and the intensity of the FIX and FIXa bands was determined using LI-COR's Image Studio software. For each blot, the fluorescence signal of the FIX and FIXa band over time was well fitted $\left(R^{2} \geq 0.97\right)$ to a single exponential, $y(t)=A e^{-k t}+y_{o}$, consistent with a pseudo-first order reaction. The concentration of FIXa at each time point was calculated based on the fluorescence signal divided by a proportionality constant $\left(y_{o} /\left[F I X_{\text {Total }}\right)\right.$. The fitting determined standard error of $y_{0}$ was propagated as the error of the concentration of FIXa in Figure 2A. The initial rate of FIXa generation was calculated as rate $=-k \times A$.

$A T$ inhibition of FIXa variants. The rate of FIXa inactivation by AT was determined by incubating $\mathrm{rFIXa}$ protein in $\mathrm{HBS}$ (20 $\mathrm{mM}$ Hepes and $150 \mathrm{mM} \mathrm{NaCl}$ ), $2 \mathrm{mM} \mathrm{CaCl}_{2}$, and $0.1 \%$ PEG8000 (Sigma-Aldrich) with or without $2 \mu \mathrm{M}$ AT (Haemtech). FIXa activity was determined using a commercial chromogenic FIXa substrate, Spectrozyme IXa (Sekisui). FIXa activity was calculated based on the rate of increase in absorbance at $405 \mathrm{~nm}$ at $37^{\circ} \mathrm{C}$. Percentage of FIXa inhibition was determined by the difference in FIXa activity between samples with and without $2 \mu \mathrm{M}$ AT divided by the FIXa activity without AT.

Activation of FX by FIXa variants. The rate of FXa generation was determined using a reconstituted intrinsic factor Xase assay as previously described with modifications $(36,50)$. First, purified FIXa protein was mixed with 75:25 phosphatidylcholine/phosphatidylserine mixture (PC/PS; Avanti) for a final reaction 
mixture concentration of $20 \mu \mathrm{M}$ in $\mathrm{HBS}, 2 \mathrm{mM} \mathrm{CaCl}_{2}$, and $0.1 \%$ PEG8000. In experiments with FVIIIa cofactor, FVIIIa was generated by mixing FVIII (Pfizer) with at least 40-fold excess thrombin (Haemtech) for 30 seconds and then added to FIXa reaction mixture containing 2-fold excess hirudin to inhibit thrombin activity. Last, plasma-derived FX (gift from S. Krishnaswamy, the Children's Hospital of Philadelphia, Philadelphia, Pennsylvania, USA) was added to the reaction mixture to initiate FXa generation. Aliquots for time course were removed and quenched by a 1:18 dilution of samples into $50 \mathrm{mM}$ EDTA-containing buffer to chelate calcium and abrogate FIXa activity.

The amount of FXa generated at each time point was determined using a commercial chromogenic FXa substrate, Spectrozyme Xa (Sekisui), according to the manufacturer's instructions. Equal amounts of quenched sample were mixed with $0.5 \mathrm{mM}$ Spectrozyme Xa in a 96-well plate (Corning), and the FXa activity was determined by measuring the rate of increase in absorbance at $405 \mathrm{~nm}$ at $37^{\circ} \mathrm{C}$. A standard curve of known FXa concentrations and FXa activity for Spectrozyme Xa was used to determine the concentration of FXa at each time point from the intrinsic Xase enzyme kinetic assay. These FXa concentrations were plotted as a function of time, and a linear fit in Microsoft Excel was used to calculate the initial rate of $\mathrm{FXa}$ generation (nM/s). Linear fits were determined with 5 to 8 points; later time points were removed if evident of a multiphasic rate.

$T G A$. For studies with rFIX, HB plasma was combined with rFIX protein, FXIa (Haemtech, final plasma concentration $12.5 \mathrm{ng} / \mathrm{mL}$ ), and 75:25 PC/PS mixture (4 $\mu \mathrm{M}$ final plasma concentration). For studies with rFIXa, HB plasma was combined only with rFIXa-mixed PC/PS. Thrombin generation reaction was initiated with the addition of $\mathrm{CaCl}_{2}$ (7.5 mM final) and the fluorogenic thrombin substrate z-Gly-Gly-ArgAMC (0.5 mM final; Bachem). The fluorescence was measured on a SpectraMax plate reader with emission and excitation wavelengths of $360 \mathrm{~nm}$ and $460 \mathrm{~nm}$, respectively. Thrombin generation was calibrated per Technoclone's instructions. Thrombograms as well as derived parameters of peak thrombin, ETP, and lag time were determined with Technoclone software.

Plasma assays with emicizumab. For plasma-based assays where emicizumab substituted for FVIIIa activity, endogenous FVIIIa activity was inhibited with a cocktail of monoclonal anti-FVIII antibodies (GMA): $50 \mu \mathrm{g} / \mathrm{mL}$ GMA-8009 and $5 \mu \mathrm{g} / \mathrm{mL}$ GMA-8021. Emicizumab was provided by Gallia G. Levy (Genentech, Inc., San Francisco, California, USA). The final plasma concentration of emicizumab was $100 \mathrm{nM}$. HB plasma was diluted less than or equal to $10 \%$ by these modifications. A separate standard curve was determined for emicizumab-substituted HB plasma.

Experiments with plasma from $H B$ gene therapy subjects expressing FIX-R338L. Subject plasma collected in sodium citrate was diluted 10 -fold with HBS. FIX activity was determined using the aPTT clotting assay as described above, with the exception that dilutions of pooled normal human plasma (George King) were used as a standard to relate clot time to FIX activity in HB plasma with and without emicizumab.

Statistics. Unless noted otherwise, curve fittings and statistical analysis were performed with OriginPro Software package. Means were compared with a 2-tailed $t$ test with $P$ values greater than or equal to 0.05 considered not significant.

Study approval. The institutional review board of the Children's Hospital of Philadelphia, Philadelphia, Pennsylvania, USA, reviewed and approved all experiments. All subjects provided informed consent before their participation in the study.

\section{Author contributions}

BJSJ designed and conducted experiments, interpreted data, and drafted the manuscript. JDF designed and conducted experiments and revised the manuscript. LAG provided clinical samples, provided guidance, and revised the manuscript. RMC and VRA designed experiments, interpreted data, provided guidance, and revised the manuscript.

\section{Acknowledgments}

We gratefully acknowledge helpful discussion from Sriram Krishnaswamy and David Lillicrap (Queen's University). We very much appreciate the gift of emicizumab from Gallia G. Levy. We also appreciate the use of plasma from HB gene therapy subjects from Katherine High (Spark Therapuetics) and John Murphy (Pfizer). BJSJ reports receiving support from the Bayer Fellowship Project Award 2015; a HTRS/Novo Nordisk 2015 Mentored Research Award in Hemophilia or Rare Bleeding Disorders from the Hemostasis and Thrombosis Research Society, Inc., which was supported by Novo Nordisk Inc.; and NIH/National Heart, Lung, and 
Blood Institute (NHLBI) 1K08HL140078. VRA reports receiving NIH/NHLBI funding P01HL64190 and P01HL139420 and support from Pfizer's Aspire competitive grants program.

Address correspondence to: Valder R. Arruda, The Children's Hospital of Philadelphia, 3501 Civic Center Blvd., 5056 Colket Translational Research Center, Philadelphia, Pennsylvania 19104, USA. Phone: 215.590.4907; Email: arruda@email.chop.edu.

1. Konkle BA, Huston H, Nakaya Fletcher S. Hemophilia B. In: Adam MP, et al. eds. GeneReviews. Seattle, Washington, USA: University of Washington; 1993.

2. Manno CS, et al. Successful transduction of liver in hemophilia by AAV-Factor IX and limitations imposed by the host immune response. Nat Med. 2006;12(3):342-347.

3. Ertl HCJ, High KA. Impact of AAV capsid-specific T-cell responses on design and outcome of clinical gene transfer trials with recombinant adeno-associated viral vectors: an evolving controversy. Hum Gene Ther. 2017;28(4):328-337.

4. Nathwani AC, et al. Long-term safety and efficacy of factor IX gene therapy in hemophilia B. N Engl J Med. 2014;371(21):1994-2004.

5. Nathwani AC, et al. Adenovirus-associated virus vector-mediated gene transfer in hemophilia B. N Engl J Med. 2011;365(25):2357-2365.

6. Samelson-Jones BJ, Arruda VR. Protein-engineered coagulation factors for hemophilia gene therapy. Mol Ther Methods Clin Dev. 2019;12:184-201.

7. Miesbach W, et al. Gene therapy with adeno-associated virus vector 5-human factor IX in adults with hemophilia B. Blood. 2018;131(9):1022-1031.

8. Rangarajan S, et al. AAV5-factor VIII gene transfer in severe hemophilia A. N Engl J Med. 2017;377(26):2519-2530.

9. Doshi BS, Arruda VR. Gene therapy for hemophilia: what does the future hold? Ther Adv Hematol. 2018;9(9):273-293.

10. George LA, et al. Hemophilia B gene therapy with a high-specific-activity factor IX variant. N Engl J Med. 2017;377(23):2215-2227.

11. Finn JD, et al. The efficacy and the risk of immunogenicity of FIX Padua (R338L) in hemophilia B dogs treated by AAV muscle gene therapy. Blood. 2012;120(23):4521-4523.

12. Cantore A, et al. Hyperfunctional coagulation factor IX improves the efficacy of gene therapy in hemophilic mice. Blood. 2012;120(23):4517-4520.

13. Crudele JM, et al. AAV liver expression of FIX-Padua prevents and eradicates FIX inhibitor without increasing thrombogenicity in hemophilia B dogs and mice. Blood. 2015;125(10):1553-1561.

14. Cantore A, et al. Liver-directed lentiviral gene therapy in a dog model of hemophilia B. Sci Transl Med. 2015;7(277):277ra28

15. Monahan PE, et al. Employing a gain-of-function factor IX variant R338L to advance the efficacy and safety of hemophilia B human gene therapy: preclinical evaluation supporting an ongoing adeno-associated virus clinical trial. Hum Gene Ther. 2015;26(2):69-81.

16. French RA, et al. Complete correction of hemophilia B phenotype by FIX-Padua skeletal muscle gene therapy in an inhibitor-prone dog model. Blood Adv. 2018;2(5):505-508.

17. Oldenburg J, et al. Emicizumab prophylaxis in hemophilia A with inhibitors. N Engl J Med. 2017;377(9):809-818.

18. Arruda VR, Doshi BS, Samelson-Jones BJ. Novel approaches to hemophilia therapy: successes and challenges. Blood. 2017;130(21):2251-2256.

19. Weyand AC, Pipe SW. New therapies for hemophilia. Blood. 2019;133(5):389-398.

20. Lenting PJ, Denis CV, Christophe OD. Emicizumab, a bispecific antibody recognizing coagulation factors IX and X: how does it actually compare to factor VIII? Blood. 2017;130(23):2463-2468.

21. Kitazawa T, et al. A bispecific antibody to factors IXa and X restores factor VIII hemostatic activity in a hemophilia A model. Nat Med. 2012;18(10):1570-1574.

22. Kitazawa T, et al. Factor VIIIa-mimetic cofactor activity of a bispecific antibody to factors IX/IXa and X/Xa, emicizumab, depends on its ability to bridge the antigens. Thromb Haemost. 2017;117(7):1348-1357.

23. Mahlangu J, et al. Emicizumab prophylaxis in patients who have hemophilia A without inhibitors. $N$ Engl J Med. 2018;379(9):811-822.

24. Zögg T, Brandstetter H. Complex assemblies of factors IX and X regulate the initiation, maintenance, and shutdown of blood coagulation. Prog Mol Biol Transl Sci. 2011;99:51-103.

25. Marder VJ, Aird WC, Bennett JS, Schulman S, White GC. Hemostasis Thrombosis: Basic Principles Clinical Practice. Philadelphia, Pennsylvania, USA: Lippincott Williams \& Wilkins; 2013.

26. van Dieijen G, Tans G, Rosing J, Hemker HC. The role of phospholipid and factor VIIIa in the activation of bovine factor X. J Biol Chem. 1981;256(7):3433-3442.

27. Di Scipio RG, Kurachi K, Davie EW. Activation of human factor IX (Christmas factor). J Clin Invest. 1978;61(6):1528-1538.

28. Osterud B, Rapaport SI. Activation of factor IX by the reaction product of tissue factor and factor VII: additional pathway for initiating blood coagulation. Proc Natl Acad Sci U S A. 1977;74(12):5260-5264.

29. White GC, Beebe A, Nielsen B. Recombinant factor IX. Thromb Haemost. 1997;78(1):261-265.

30. Simioni P, et al. X-linked thrombophilia with a mutant factor IX (factor IX Padua). N Engl J Med. 2009;361(17):1671-1675.

31. Muczynski V, Christophe OD, Denis CV, Lenting PJ. Emerging therapeutic strategies in the treatment of hemophilia A. Semin Thromb Hemost. 2017;43(6):581-590.

32. Westmark PR, Tanratana P, Sheehan JP. Selective disruption of heparin and antithrombin-mediated regulation of human factor IX. J Thromb Haemost. 2015;13(6):1053-1063.

33. Milanov P, et al. Engineered factor IX variants bypass FVIII and correct hemophilia A phenotype in mice. Blood. 2012;119(2):602-611. 
34. Quade-Lyssy P, et al. Next generation FIX muteins with FVIII-independent activity for alternative treatment of hemophilia A. J Thromb Haemost. 2014;12(11):1861-1873.

35. Yuan QP, Walke EN, Sheehan JP. The factor IXa heparin-binding exosite is a cofactor interactive site: mechanism for antithrombin-independent inhibition of intrinsic tenase by heparin. Biochemistry. 2005;44(9):3615-3625.

36. Chang J, et al. Changing residue 338 in human factor IX from arginine to alanine causes an increase in catalytic activity. $J$ Biol Chem. 1998;273(20):12089-12094.

37. Perot E, et al. Expression and characterization of a novel human recombinant factor IX molecule with enhanced in vitro and in vivo clotting activity. Thromb Res. 2015;135(5):1017-1024.

38. MacFarlane RG. An enzyme cascade in the blood-clotting mechanism, and its function as a biochemical amplifier. Nature. 1964;202:498-499.

39. Persson E, Bak H, Olsen OH. Substitution of valine for leucine 305 in factor VIIa increases the intrinsic enzymatic activity J Biol Chem. 2001;276(31):29195-29199.

40. Kao CY, Yang SJ, Tao MH, Jeng YM, Yu IS, Lin SW. Incorporation of the factor IX Padua mutation into FIX-Triple improves clotting activity in vitro and in vivo. Thromb Haemost. 2013;110(2):244-256.

41. Schmaier AH, et al. Protease nexin-2/amyloid $\beta$ protein precursor. A tight-binding inhibitor of coagulation factor IXa. J Clin Invest. 1993;92(5):2540-2545.

42. Samis JA, Kam E, Nesheim ME, Giles AR. Neutrophil elastase cleavage of human factor IX generates an activated factor IX-like product devoid of coagulant function. Blood. 1998;92(4):1287-1296.

43. Minami H, Nogami K, Soeda T, Kitazawa T, Hattori K, Shima M. The factor VIII heavy chain improves emicizumab-tenase assembly to enhance the factor VIII-mimicking cofactor activity. Thromb Res. 2018;166:77-79.

44. Kolkman JA, Lenting PJ, Mertens K. Regions 301-303 and 333-339 in the catalytic domain of blood coagulation factor IX are factor VIII-interactive sites involved in stimulation of enzyme activity. Biochem J. 1999;339(pt 2):217-221.

45. Jenkins PV, Freas J, Schmidt KM, Zhou Q, Fay PJ. Mutations associated with hemophilia A in the 558-565 loop of the factor VIIIa A2 subunit alter the catalytic activity of the factor Xase complex. Blood. 2002;100(2):501-508.

46. Kristensen LH, Olsen OH, Blouse GE, Brandstetter H. Releasing the brakes in coagulation Factor IXa by co-operative maturation of the substrate-binding site. Biochem J. 2016;473(15):2395-2411.

47. Bottema CD, et al. The pattern of spontaneous germ-line mutation: relative rates of mutation at or near CpG dinucleotides in the factor IX gene. Hum Genet. 1993;91(5):496-503.

48. Sun YM, Jin DY, Camire RM, Stafford DW. Vitamin K epoxide reductase significantly improves carboxylation in a cell line overexpressing factor X. Blood. 2005;106(12):3811-3815.

49. Arruda VR, et al. Posttranslational modifications of recombinant myotube-synthesized human factor IX. Blood. 2001;97(1):130-138

50. Toso R, Zhu H, Camire RM. The conformational switch from the factor X zymogen to protease state mediates exosite expression and prothrombinase assembly. J Biol Chem. 2008;283(27):18627-18635. 